\title{
The Heine-Stieltjes correspondence and a new angular momentum projection for many-particle systems
}

\author{
Feng Pan, ${ }^{1,2,3}$ Bo Li, ${ }^{1}$ Yao-Zhong Zhang, ${ }^{3}$ and Jerry P. Draayer ${ }^{2}$ \\ ${ }^{1}$ Department of Physics, Liaoning Normal University, Dalian 116029, China \\ ${ }^{2}$ Department of Physics and Astronomy, Louisiana State University, Baton Rouge, LA 70803-4001, USA \\ ${ }^{3}$ School of Mathematics and Physics, The University of Queensland, Brisbane, Qld 4072, Australia
}

(Dated: May 29, 2022)

\begin{abstract}
A new angular momentum projection for systems of particles with arbitrary spins is formulated based on the Heine-Stieltjes correspondence, which can be regarded as the solutions of the mean-field plus pairing model in the strong pairing interaction $G \rightarrow \infty$ limit. Properties of the Stieltjes zeros of the extended Heine-Stieltjes polynomials, of which the roots determine the projected states, and the related Van Vleck zeros are discussed. The electrostatic interpretation of these zeros is presented. As examples, applications to $n$ nonidentical particles of spin- $1 / 2$ and to identical bosons or fermions are made to elucidate the procedure and properties of the Stieltjes zeros and the related Van Vleck zeros. It is shown that the new angular momentum projection for $n$ identical bosons or fermions can be simplified with the branching multiplicity formula of $U(N) \downarrow O(3)$ and the special choices of the parameters used in the projection. Especially, it is shown that the solutions for identical bosons can always be expressed in terms of zeros of Jacobi polynomials. However, unlike non-identical particle systems, the $n$-coupled states of identical particles are non-orthogonal with respect to the multiplicity label after the projection.
\end{abstract}

PACS numbers: 21.60.Cs, 21.60.Fw, 03.65.Fd, 02.20.Qs, 02.30.Ik

\section{INTRODUCTION}

The angular momentum projection or construction of many-body wavefunctions with definite total angular momentum from a set of single-particle product states has practical value in quantum many-body physics [1-3]. For a few particle systems, the Clebsch-Gordan (CG) coefficients, 3j symbols or Wigner coefficients, can be used straightforwardly for this purpose. However, with increasing particle numbers, the CG couplings become tedious and cumbersome because with increasing particle numbers, the number of intermediate angular momentum quantum numbers the are required to label different states with the same total angular momentum grows combinatorially. In practical applications, the projection technique of Löwdin has been one of the most popular [1]. This method uses the angular momentum projection operator to project a set of single-particle product states into states with definite total angular momentum, which requires solution of the eigenvalue problem of the projection operator matrix constructed from the relevant single-particle product states. In [3], Biedenharn and Louck proposed the Wigner operator method that combines CG couplings with results from the theory of the symmetric groups. However, their method can only be worked out explicitly for $n$ nonidentical particles of spin-1/2. In the case of the nuclear shell model, other procedures are used to construct states with definite total angular momentum quantum number $J$. One, called the $M$ scheme, starts with single-particle product states with good total angular momentum projection and utilizes a simple subtraction procedure to extract states with good total angular momentum [4], and another uses direct angular momentum couplings and is usually referred to as the $J$-coupled scheme for identical particles or the JT-coupled scheme when applied to a protonneutron system [5]. Alternatively, the projection operator constructed in terms of an integration of the product of the rotational group element and its matrix element (Wigner's D-function) of a given angular momentum over the Euler angles can also be used [2], as or example, in construction of Elliott basis [6] of $S U(3) \supset S O(3)$ and in the projected shell model calculations [7]. These methods can all be relatively easily implemented in computer codes designed for their respective purpose. Their drawbacks lie in the fact that much CPU time is needed when the dimension of the subspace spanned by the relevant single-particle product states is really large, especially when the projection operator is constructed in terms of an integration of the product of the rotational group element and its matrix element of a given angular momentum over the Euler angles is used because the WheelerHill integral involved is difficult to be treated accurately in the code.

Recently, it has been shown that the angular momentum projection may be realized by solving a set of Bethe ansatz equations $[8,9]$. The purpose of this work is to show that the Bethe ansatz equations can be solved relatively easily from zeros of the associated extended Heine-Stieltjes polynomials from the Heine-Stieltjes correspondence [9-14]. In Sec. II, we will revisit the Bethe ansatz method for the angular momentum projection. The Heine-Stieltjes correspondence related to the problem, together with properties of the Heine-Stieltjes polynomials and their electrostatic interpretation, will be 
studied in Sec. III. As an examples, the application to $n$ nonidentical particles with spin- $1 / 2$ will be shown in Sec. IV, which is also related to the eigenvalue problem of the pure pairing interactions among valence nucleon pairs over a set of deformed Nilsson orbits, while applications to systems of identical bosons and fermions will be discussed in Sec. V. A brief summary will be given in Sec. VI.

\section{THE BETHE ANSATZ METHOD FOR ANGULAR MOMENTUM PROJECTION}

Let $\left\{J_{\mu}^{\alpha} ; \mu=+,-, 0\right\}$, where $\alpha=1,2, \cdots, n$, be generators of the $\alpha$-th copy of the $S U(2)$ algebra, which satisfy the commutation relations:

$$
\begin{aligned}
& {\left[J_{+}^{\alpha}, J_{-}^{\beta}\right]=2 \delta_{\alpha \beta} J_{0}^{\alpha},} \\
& {\left[J_{0}^{\alpha}, J_{ \pm}^{\beta}\right]= \pm \delta_{\alpha \beta} J_{ \pm}^{\alpha},}
\end{aligned}
$$

and $\left|j_{\alpha}, m_{\alpha}\right\rangle$ be the corresponding orthonormal basis vectors with angular momentum quantum number $j_{\alpha}$ and quantum number $m_{\alpha}$ of its third component. According to the Bethe ansatz method, one can write $n$-coupled state with total angular momentum $J=\sum_{\alpha} j_{\alpha}-k$ and $M=J$ as

$$
\left.\mid \zeta ; J, M=J)=J_{-}\left(x_{1}^{(\zeta)}\right) J_{-}\left(x_{2}^{(\zeta)}\right) \cdots J_{-}\left(x_{k}^{(\zeta)}\right) \mid \text { h.w. }\right\rangle,
$$

where $\mid$ h.w. $\rangle=\prod_{\alpha=1}^{n}\left|j_{\alpha}, m_{\alpha}=j_{\alpha}\right\rangle$ is the $\mathrm{SU}(2)$ highest weight state satisfying $J_{+}^{\alpha} \mid$ h.w. $\rangle=0$ for any $\alpha$,

$$
J_{-}\left(x_{i}^{(\zeta)}\right)=\sum_{\alpha=1}^{n} \frac{1}{x_{i}^{(\zeta)}-\epsilon_{\alpha}} J_{-}^{\alpha},
$$

in which the parameters $\left\{\epsilon_{\alpha}\right\}$ can be any set of unequal numbers, and $\zeta$ is used to distinguish different $n$-coupled states with the same angular momentum $J$. Because (2) is the highest weight state of the angular momentum $J$, it should satisfy the condition

$$
\begin{gathered}
\left.J_{+} \mid \zeta ; J, M=J\right)= \\
\left.J_{+} J_{-}\left(x_{1}^{(\zeta)}\right) J_{-}\left(x_{2}^{(\zeta)}\right) \cdots J_{-}\left(x_{k}^{(\zeta)}\right) \mid \text { h.w. }\right\rangle=0,
\end{gathered}
$$

where $J_{+}=\sum_{\alpha} J_{+}^{\alpha}$. By using the commutation relations (1), (4) requires that the Bethe ansatz equations (BAEs)

$$
\sum_{\alpha=1}^{n} \frac{2 j_{\alpha}}{x_{i}^{(\zeta)}-\epsilon_{\alpha}}-\sum_{t=1(\neq i)}^{k} \frac{2}{x_{i}^{(\zeta)}-x_{t}^{(\zeta)}}=0
$$

must be satisfied for $i=1,2, \cdots, k$. It is clear that the multiplicity label $\zeta=1,2, \cdots, d(n, k)$ in $(2)$ is taken to be the label of different solutions $\left\{x^{(\zeta)}\right\}$ of Eq. (5). It can be verified $[8,9]$ that the number of solutions $d(n, k)$ of Eq. (5) equals exactly to the multiplicity in the reduction $j_{1} \otimes j_{2} \otimes \cdots \otimes j_{n} \downarrow J$, which can be calculated by

$$
d(n, k)=\eta(n, k)-\sum_{\mu=0}^{k-1} d(n, \mu)
$$

where

$$
\eta(n, k)=\sum_{\mu_{1}=0}^{2 j_{1}} \cdots \sum_{\mu_{n}=0}^{2 j_{n}} \delta_{q, k}
$$

in which $q=\sum_{i=1}^{n} \mu_{i}$. From Eqs. (6) and (7), the multiplicity $d(n, k)$ can be calculated recursively from $d(n, 0)=1$.

Once the solutions $\left\{x_{1}^{(\zeta)}, \cdots, x_{k}^{(\zeta)}\right\}$ are obtained from Eq. (5), the $n$-coupled state with any $M$ can be expressed in the standard way as

$$
\left.\mid \zeta ; J, M)=\sqrt{\frac{(J+M) !}{(2 J) !(J-M) !}} J_{-}^{J-M} \mid \zeta ; J, J\right),
$$

where $\mid \zeta ; J, J)$ is given by Eq. (2).

Because the uncoupled basis vectors $\left\{\left|j_{\alpha}, m_{\alpha}\right\rangle\right\}$ are orthonormal, substituting (3) into (2), one can find that the unnormalized angular momentum multi-coupling coefficient is given by

$$
\begin{aligned}
& \left(j_{1}, j_{1}-\mu_{1} ; \cdots ; j_{n}, j_{n}-\mu_{n} \mid \zeta ; J, J\right)= \\
& S^{(k)}\left(\beta_{1}^{\mu_{1}}, \cdots, \beta_{n}^{\mu_{n}}\right) \prod_{i=1}^{n} \sqrt{\frac{\left(2 j_{i}\right) ! \mu_{i} !}{\left(2 j_{i}-\mu_{n}\right) !}}
\end{aligned}
$$

where the condition $\sum_{\alpha}^{n} \mu_{\alpha}=k$ must be satisfied, $S^{(k)}\left(\beta_{1}^{\mu_{1}}, \cdots, \beta_{n}^{\mu_{n}}\right)$ is the $k \times n$-variable symmetric function, in which $\beta_{\alpha}^{\mu_{\alpha}}$ is the shorthand notation of taken $\mu_{\alpha}$ variables $\left\{\beta_{i_{1}, \alpha}, \cdots, \beta_{i_{\mu_{\alpha}}, \alpha}\right\}$ with $i_{1} \neq i_{2} \neq \cdots \neq i_{\mu_{\alpha}}$ from $\left\{\beta_{1, \alpha}, \cdots, \beta_{k, \alpha}\right\}$, and

$$
\beta_{i, \alpha}=\frac{1}{x_{i}^{(\zeta)}-\epsilon_{\alpha}}
$$

When $n=2$ and $k=2$, for example, we have $S^{(2)}\left(\beta_{1}^{2}\right)=\beta_{11} \beta_{12}, S^{(2)}\left(\beta_{1}, \beta_{2}\right)=\beta_{11} \beta_{22}+\beta_{21} \beta_{12}$, and $S^{(2)}\left(\beta_{2}^{2}\right)=\beta_{12} \beta_{22}$. The normalized angular momentum multi-coupling coefficient is

$$
\begin{gathered}
\left\langle j_{1}, j_{1}-\mu_{1}, \cdots, j_{n}, j_{n}-\mu_{n} \mid \zeta ; J, J\right\rangle= \\
\left(j_{1}, j_{1}-\mu_{1}, \cdots, j_{n}, j_{n}-\mu_{n} \mid \zeta ; J, J\right) / \mathcal{N}
\end{gathered}
$$

where

$$
\mathcal{N}=\left(\sum_{\mu_{1} \ldots \mu_{n}}\left(j_{1}, j_{1}-\mu_{1}, \cdots, j_{n}, j_{n}-\mu_{n} \mid \zeta ; J, J\right)^{2}\right)^{\frac{1}{2}}
$$


in which the summation should be restricted by $\sum_{\alpha}^{n} \mu_{\alpha}=$ $k$.

In comparison to the traditional projection methods $[1,2,4,5]$, the Bethe ansatz method for angular momentum projection is more efficient, which only needs to solve $k$-coupled algebraic BAEs. However, one must solve $d(n, k)$-dimensional matrix eigenvalue problem in the traditional projection methods. The dimension $d(n, k)$ increases with the increasing of $n$ and $k$ in a non-polynomial way as shown in (6). Therefore, the Bethe ansatz method for angular momentum projection is advantageous if there is a simple way to solve the BAEs (5).

\section{THE HEINE-STIELTJES CORRESPONDENCE}

It has been shown that the BAEs similar to those shown in (5) may be solved from zeros of the corresponding extended Heine-Stieltjes polynomials [9-14]. Through the Heine-Stieltjes correspondence [9, 10], for the BAEs (5), one may consider the following secondorder Fuchsian equation:

$$
A_{n}(x) y_{k}^{\prime \prime}(x)+B_{n-1}(x) y_{k}^{\prime}(x)-V_{n-2}(x) y_{k}(x)=0,
$$

where $A_{n}(x)=\prod_{\alpha=1}^{n}\left(x-\epsilon_{\alpha}\right)$ is a polynomial of degree $n$, the polynomial $B_{n-1}(x)$ is given as

$$
B_{n-1}(x) / A_{n}(x)=-\sum_{\alpha=1}^{n} \frac{2 j_{\alpha}}{x-\epsilon_{\alpha}},
$$

and $V_{n-2}(x)$ is called Van Vleck polynomial [15] of degree $n-2$, which are determined according to Eq. (13). Let $\left\{x_{i}, i=1,2, \cdots, k\right\}$ be zeros of the extended HeineStieltjes polynomial $y_{k}(x)$, which are often called Stieltjes zeros. We may write $y_{k}(x)=\prod_{i=1}^{k}\left(x-x_{i}\right)$. At any zero $x_{i}$ of $y_{k}(x)$, there is the identity

$$
\frac{y_{k}^{\prime \prime}\left(x_{i}\right)}{y_{k}^{\prime}\left(x_{i}\right)}=\sum_{t=1(t \neq i)}^{k} \frac{2}{x_{i}-x_{t}} .
$$

It is obvious that, at any zero $x_{i}$ of $y_{k}(x),(13)$ results in the BAEs (5). Generally, we also have

$$
\begin{gathered}
\frac{y_{k}^{\prime \prime}(x)}{y_{k}(x)}=\sum_{1 \leq i<t \leq k} \frac{2}{\left(x-x_{i}\right)\left(x-x_{t}\right)}= \\
\sum_{1 \leq i \neq t \leq k} \frac{2}{x-x_{i}} \frac{1}{\left(x_{i}-x_{t}\right)}, \\
\frac{y_{k}^{\prime}(x)}{y_{k}(x)}=\sum_{i=1}^{k} \frac{1}{x-x_{i}} .
\end{gathered}
$$

Substituting (16) and (17) into (13), we have

$$
\begin{gathered}
V_{n-2}^{(\zeta)}(x)=A_{n}(x) \times \\
\sum_{i=1}^{k} \frac{1}{x-x_{i}^{(\zeta)}}\left(\sum_{t \neq i} \frac{2}{x_{i}^{(\zeta)}-x_{t}^{(\zeta)}}-\sum_{\alpha} \frac{2 j_{\alpha}}{x-\epsilon_{\alpha}}\right) .
\end{gathered}
$$

By using the BAEs (5), (18) becomes

$$
V_{n-2}^{(\zeta)}(x)=A_{n}(x) \sum_{\alpha=1}^{n} \frac{1}{x-\epsilon_{\alpha}}\left(\sum_{i=1}^{k} \frac{2 j_{\alpha}}{x_{i}^{(\zeta)}-\epsilon_{\alpha}}\right) .
$$

Eq. (19) shows that zeros $\left\{\bar{x}_{l}^{(\zeta)} ; l=1,2, \cdots, n-2\right\}$ of the Van Vleck polynomial $V_{n-2}^{(\zeta)}(x)$ related to the $\zeta$-th extended Heine-Stieltjes polynomial $y_{k}^{(\zeta)}(x)=\prod_{i=1}^{k}(x-$ $\left.x_{i}^{(\zeta)}\right)$ are determined by

$$
\sum_{\alpha=1}^{n} \frac{1}{\bar{x}_{l}^{(\zeta)}-\epsilon_{\alpha}}\left(\sum_{i=1}^{k} \frac{2 j_{\alpha}}{\epsilon_{\alpha}-x_{i}^{(\zeta)}}\right)=0 .
$$

$\left\{\bar{x}_{l}^{(\zeta)} ; l=1,2, \cdots, n-2\right\}$ are called Ven Vleck zeros related to the $\zeta$-th extended Heine-Stieltjes polynomial $y_{k}^{(\zeta)}(x)$. Once the Van Vleck zeros are obtained from Eq. (20), $V_{n-2}^{(\zeta)}(x)$ can be expressed explicitly as

$$
V_{n-2}^{(\zeta)}(x)=c_{n, k} \prod_{l=1}^{n-2}\left(x-\bar{x}_{l}^{(\zeta)}\right),
$$

where $c_{n, k}$ is a constant depending on $n, k$, and the parameters $\epsilon_{\alpha}(\alpha=1, \cdots, n)$.

If $\epsilon_{\alpha}(\alpha=1, \cdots, n)$ are chosen to be real, according to the Stieltjes results [15], the electrostatic interpretation of the location of zeros of the extended Heine-Stieltjes polynomial $y_{k}(x)$ may be stated as follows. Put $n$ negative fixed charges $-j_{\alpha}$ at $\epsilon_{\alpha}$ for $\alpha=1, \cdots, n$ along a real line, respectively, and allow $k$ positive unit charges to move freely on the two dimensional complex plane. Therefore, up to a constant, the total energy functional may be written as

$$
\begin{gathered}
U\left(x_{1}, x_{2}, \cdots, x_{k}\right)= \\
\sum_{i=1}^{k} \sum_{\alpha}^{n} j_{\alpha} \ln \left|x_{i}-\epsilon_{\alpha}\right|-\sum_{1 \leq i \neq t \leq k} \ln \left|x_{i}-x_{t}\right| .
\end{gathered}
$$

The BAEs given in Eq. (5) imply that there are $d(n, k)$ different configurations for the position of the $k$ positive 
charges $\left\{x_{1}^{(\zeta)}, \cdots, x_{k}^{(\zeta)}\right\}$ with $\zeta=1,2, \cdots, d(n, k)$, corresponding to global minimums of the total energy.

Similarly, let

$$
\rho_{\alpha}^{(\zeta)}(k)=2 j_{\alpha} \sum_{i=1}^{k} \frac{1}{\epsilon_{\alpha}-x_{i}^{(\zeta)}}
$$

which is now called Van Vleck charges related to the zeros of the $\zeta$-th extended Heine-Stieltjes polynomial $y_{k}^{(\zeta)}(x)$. Put $n$ Van Vleck charges $\rho_{\alpha}^{(\zeta)}(k)$ at positions $\epsilon_{\alpha}$ for $\alpha=1, \cdots, n$ along a real line, respectively, and allow one unit charge to move freely on the two dimensional complex plane. Eq. (20) provides $n-2$ possible equilibrium positions $\left\{\bar{x}_{l}^{(\zeta)} ; l=1,2, \cdots, n-2\right\}$ of the unit moving charge for the electrostatic system.

Let

$$
\Lambda(x)=\frac{y_{k}^{\prime}(x)}{y_{k}(x)}=\sum_{i=1}^{k} \frac{1}{x-x_{i}} .
$$

As shown in [16], $\Lambda(x)$ satisfies the Riccati type equation

$$
\Lambda^{\prime}(x)+\Lambda^{2}(x)+\sum_{i=1}^{k} \sum_{\alpha}^{n} \frac{2 j_{\alpha}}{\left(x-x_{i}\right)\left(\epsilon_{\alpha}-x_{i}\right)}=0
$$

in this case. The Van Vleck charges $\rho_{\alpha}$ can be expressed as

$$
\rho_{\alpha}=2 j_{\alpha} \Lambda\left(\epsilon_{\alpha}\right)
$$

There are a series of high order differential equations [16] for $\Lambda\left(\epsilon_{\alpha}\right)$. For example, the lowest order one is

$$
\begin{gathered}
\left(1-2 j_{\beta}\right) \Lambda^{\prime}\left(\epsilon_{\beta}\right)+\Lambda^{2}\left(\epsilon_{\beta}\right) \\
+\sum_{\alpha \neq \beta} 2 j_{\alpha} \frac{\Lambda\left(\epsilon_{\beta}\right)-\Lambda\left(\epsilon_{\alpha}\right)}{\epsilon_{\alpha}-\epsilon_{\beta}}=0 .
\end{gathered}
$$

It seems that the solutions of $\Lambda\left(\epsilon_{\alpha}\right)$ of Eq. (27) or from a series of high order differential equations can be used to determine Van Vleck zeros, and eventually solve the BAEs (5) as shown in [16]. It should be noted that the solutions of $\left\{\Lambda\left(\epsilon_{\alpha}\right)\right\}$ from those Riccati type equations only depend on the parameters $\left\{\epsilon_{\alpha}\right\}$ and $\left\{j_{\alpha}\right\}$, but do not explicitly depend on $k$ and $\zeta$. Therefore, the solutions of $\left\{\Lambda\left(\epsilon_{\alpha}\right)\right\}$ from those Riccati type equations are numerous. One should try to search for a set of solution $\left\{\Lambda\left(\epsilon_{\alpha}\right)\right\}$ corresponding to specific $k$ and $\zeta$ from solutions with all possible $k$ and $\zeta$ obtained from Eq. (27), which explains why the other expressions of $\left\{\Lambda\left(\epsilon_{\alpha}\right)\right\}$ in terms of symmetric functions of $\left\{x_{1}, \cdots, x_{k}\right\}$ should also be used to solve the problem [16].

In order to avoid the previous mentioned ambiguity, in the following, we insist on using the method outlined in $[10]$. We write

$$
y_{k}(x)=\sum_{j=0}^{k} a_{j} x^{j}, \quad V_{n-2}(x)=\sum_{j=0}^{n-2} b_{j} x^{j},
$$

where $\left\{a_{j}\right\}$ and $\left\{b_{j}\right\}$ are the expansion coefficients to be determined. Substitution of (28) into (13) yields two matrix equations. By solving these two matrix equations, we can obtain the solutions of $\left\{a_{j}\right\}$ and $\left\{b_{j}\right\}$ for given $k$. Since there is freedom to choose the parameters $\left\{\epsilon_{\alpha} ; \alpha=\right.$ $1, \cdots, n\}$, we find the following parameter settings to be a simple and convenient choice due to the fact that there is an additional reflection symmetry in (5):

$$
\left\{\begin{array}{l}
\epsilon_{\alpha}=-(p+1-\alpha) \text { for } \alpha \leq p, \\
\epsilon_{\alpha+p}=\alpha \text { for } \alpha \geq 1,
\end{array}\right.
$$

when $n=2 p$, and

$$
\left\{\begin{array}{l}
\epsilon_{\alpha}=-(p+1-\alpha) \text { for } \alpha \leq p, \\
\epsilon_{p+1}=0, \\
\epsilon_{\alpha+p+1}=\alpha \text { for } \alpha \geq 1,
\end{array}\right.
$$

when $n=2 p+1$. With such a choice, in addition to the $S_{k}$ permutation symmetry among indices $i=1, \cdots, k$ of $\left\{x_{1}, \cdots, x_{k}\right\}$, the Stieltjes zeros $\left\{x_{i}\right\}$ have the following additional reflection symmetries: (i) If $\left\{x_{1}, \cdots, x_{k}\right\}$ is a set of Stieltjes zeros, $\left\{-x_{1}, \cdots,-x_{k}\right\}$ is another set. (ii) When $n$ is even, there are many sets of solutions with $\left\{x_{1}=-x_{2}, x_{3}=-x_{4}, \cdots, x_{k-1}=-x_{k}\right\}$ when $k$ is even, and $\left\{x_{1}=-x_{2}, x_{3}=-x_{4}, \cdots, x_{k-2}=-x_{k-1}, x_{k}=0\right\}$ when $k$ is odd. When $n$ is odd, there are many sets of solutions with $\left\{x_{1}=-x_{2}, x_{3}=-x_{4}, \cdots, x_{k-1}=-x_{k}\right\}$ when $k$ is even. Solutions satisfying property (ii) are selfreflectional. Property (i) is strong, namely such pairs of solutions always exist, which is obvious with the substitutions of $x_{i}$ with $-x_{i}$ in Eq. (5) for $i=1, \cdots, k$. However, property (ii) only applies to a subset of solutions, namely there are other sets of solutions which may not follow property (ii). One can verify that the substitution of $\left\{x_{1}=-x_{2}, x_{3}=-x_{4}, \cdots, x_{k-1}=-x_{k}\right\}$ for $k$ even or $\left\{x_{1}=-x_{2}, x_{3}=-x_{4}, \cdots, x_{k-2}=-x_{k-1}, x_{k}=0\right\}$ for $k$ odd into Eq. (5) indeed yields $k$ consistent equations when $n$ is even, which implies that $\left\{x_{1}=-x_{2}, x_{3}=\right.$ $\left.-x_{4}, \cdots, x_{k-1}=-x_{k}\right\}$ for $k$ even or $\left\{x_{1}=-x_{2}, x_{3}=\right.$ $\left.-x_{4}, \cdots, x_{k-2}=-x_{k-1}, x_{k}=0\right\}$ for $k$ odd is possible solutions when $n$ is even. For odd $n$ cases, self-reflectional solutions only exist when $k$ is even. Because the parameters chosen satisfy the interlacing condition $\epsilon_{1}<\cdots<\epsilon_{n}$, zeros of $y_{k}(x)$ may be arranged to satisfy the interlacing condition, $\boldsymbol{\operatorname { R e }}\left(x_{1}\right) \leq \boldsymbol{\operatorname { R e }}\left(x_{2}\right) \leq \cdots \leq \boldsymbol{\operatorname { R e }}\left(x_{k}\right)$, where 
$\boldsymbol{\operatorname { R e }}\left(x_{i}\right)$ lies in one of the $n-1$ intervals $\left(\epsilon_{1}, \epsilon_{2}\right), \cdots$, $\left(\epsilon_{n-1}, \epsilon_{n}\right)$, in which the equality is only possible when the adjacent zeros are complex conjugate with each other. When two zeros are conjugate with each other with $x_{i}=x_{i+1}^{*}$, it is obvious that $\boldsymbol{\operatorname { R e }}\left(x_{i}\right)$ and $\boldsymbol{\operatorname { R e }}\left(x_{i+1}\right)$ are in the same interval $\left(\epsilon_{\alpha}, \epsilon_{\alpha+1}\right)$. The number of different such allowed configurations gives the possible solutions of $y_{k}(x)$ and the corresponding $V_{n-2}(x)$. Therefore, these properties are much helpful to simplify the problem and in search for solutions of (5).

\section{APPLICATION TO SYSTEMS WITH NONIDENTICAL SPIN-1/2 PARTICLES}

Generally, the Bethe ansatz method for angular momentum projection with the Heine-Stieltjes correspondence shown in previous sections can be applied to construct state with definite angular momentum from a set of uncoupled single-particle states of both nonidentical- and identical-particle systems. Because identical-particle systems have additional permutation symmetries, namely symmetric among identical bosons or antisymmetric among identical fermions with respect to the single-particle coordinate permutations, the procedure outlined in previous sections can be simplified. Such simplifications and applications will be shown in the next section. In this section, we only focus on a nonidenticalparticle case, in which we strictly follow the method described previously because no further simplification can be made for nonidentical-particle systems.

As the simplest but nontrivial example, we consider $n$ nonidentical particles of spin- $1 / 2$, which was previously studied by Louck and Biedenharn using the pattern calculus with the Yamanocchi symbol of an irrep of $S_{n}$ as the upper pattern used to label the outer multiplicity of $S U(2) \times \cdots \times S U(2) \downarrow S U(2)$, and the $S U(2)$ basis of the same irrep as the lower pattern [3, 17]. This is the only case that can be solved analytically by using the Wigner operator method. However, as shown in [3], the construction of coupled state with definite angular momentum for nonidentical particles of arbitrary spin can never be expressed analytically by using the pattern calculus, principally because of unsolved problems relating to the upper patterns. Specifically, by using the pattern calculus, the $n$-coupled state with total angular momentum $J$ of spin-1/2 system may be written as $[3,17]$

$$
\begin{gathered}
\left|\left(i_{1} \cdots i_{n}\right) ; J M\right\rangle= \\
\sum_{k_{1} \cdots k_{n}}\left\langle\begin{array}{cc}
2 J & 0 \\
J+M
\end{array}\left|\left\langle\begin{array}{cc}
i_{1} \\
1 & 0 \\
k_{n}
\end{array}\right) \cdots\left\langle\begin{array}{rr}
i_{n} \\
1 & 0 \\
k_{1}
\end{array}\right)\right| \begin{array}{cc}
0 & 0 \\
0
\end{array}\right\rangle \times
\end{gathered}
$$

$$
\prod_{i=1}^{n}\left|\frac{1}{2}, k_{i}-\frac{1}{2}\right\rangle
$$

where $\left(i_{1} \cdots i_{n}\right)$ with $i_{s}=0$ or 1 for $s=1, \cdots, n$, is used as the multiplicity label, the sum should be restricted with $k_{i}=0$ or 1 for $i=1, \cdots, n$, and the expansion coefficient

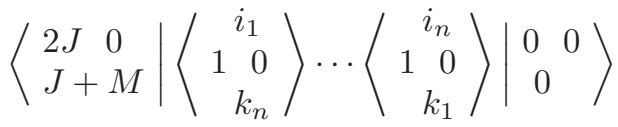

should be calculated consecutively with

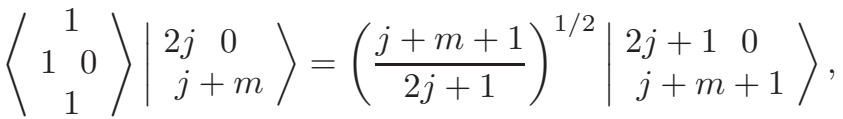

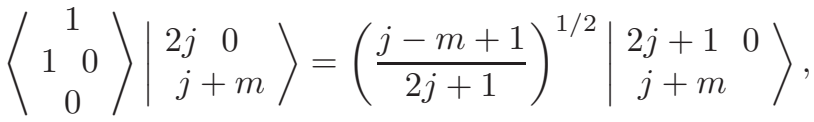

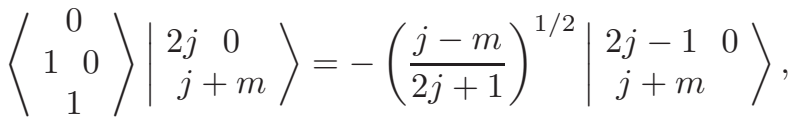

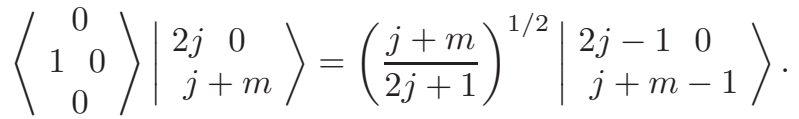

Though the expression of the expansion coefficients shown by (32) is analytic, the evaluation of (32) according to the rules shown in (33) is still cumbersome, especially the coefficients for many permissible upper patterns $\left(i_{1} \cdots i_{n}\right)$ that may lie in the null space are zero, which, however, can not be ruled out beforehand. This is the main drawback in using the upper pattern to resolve the outer multiplicity problem of unitary groups [18]. In contrast, roots of the BAEs (5) provide with all possible coupled states with the same angular momentum $J$ as shown by (2) and (3), which are mutually orthogonal with respect to the multiplicity label $\zeta$. Solutions of (5) can be obtained from Eq. (13) with the explicit expressions shown in (28). Moreover, the new angular momentum projection method outlined in Sec. II is not restricted to systems consisting of particles with the same spin, but can be applied to systems consisting of particles with arbitrary spins.

The above example is closely related to the construction of eigenstates of the pure pairing Hamiltonian in the deformed Nilsson basis with

$$
\hat{H}_{\mathrm{S}}=-G S^{+} S^{-} \text {, }
$$


where $S^{+}=\sum_{\mu} S_{\mu}^{+}=\sum_{\mu} a_{\mu \uparrow}^{\dagger} a_{\mu \downarrow}^{\dagger}$ and $S^{-}=\left(S^{+}\right)^{\dagger}$, in which $S_{\mu}^{+}=a_{\mu \uparrow}^{\dagger} a_{\mu \downarrow}^{\dagger}\left(S_{\mu}^{-}=a_{\mu \downarrow} a_{\mu \uparrow}\right)$ are pair creation (annihilation) operators. The up and down arrows in these expressions refer to time-reversed states. For simplicity, we only consider the seniority zero cases. The eigenstates of (34) can be constructed in the following way [19]: Since each Nilsson level can be occupied at most by one pair due to the Pauli principle, the local states can be regarded as quasi-spin- $1 / 2$ states. $\left|\frac{1}{2}, \frac{1}{2}\right\rangle$ stands for one pair state, while $\left|\frac{1}{2},-\frac{1}{2}\right\rangle$ stands for no pair state. Then, similar to (2), any allowed total quasi-spin $S$ and $M_{S}=S$ state of $p$ pairs over $n$ Nilsson levels can be written as

$$
\left.\left|\zeta ; S, M_{S}=S\right\rangle=\mathcal{N} S^{-}\left(x_{1}^{(\zeta)}\right) \cdots S^{-}\left(x_{t}^{(\zeta)}\right) \mid \text { h.w. }\right\rangle
$$

with $p=n-t$ pairs, where $\mathcal{N}$ is the normalization constant defined by (12), $S=n / 2-t$, |h.w. $\rangle \equiv$ $\left|\frac{1}{2}, \frac{1}{2} ; \cdots ; \frac{1}{2}, \frac{1}{2}\right\rangle$ is the product of $n$ copies of local state with highest weight of quasi-spin- $1 / 2$, and

$$
S^{-}\left(x_{i}^{(\zeta)}\right)=\sum_{\mu=1}^{n} \frac{1}{x_{i}^{(\zeta)}-\epsilon_{\mu}} S_{\mu}^{-},
$$

in which the parameters $\left\{\epsilon_{\mu}\right\}$ can be any set of unequal numbers, and $\zeta$ is used to distinguish different $n$ coupled states with the same quasi-spin $S$. The variables $\left\{x_{1}^{(\zeta)}, \cdots, x_{t}^{(\zeta)}\right\}$ should satisfy

$$
\sum_{\mu=1}^{n} \frac{1}{x_{i}^{(\zeta)}-\epsilon_{\mu}}-\sum_{l=1(\neq i)}^{t} \frac{2}{x_{i}^{(\zeta)}-x_{l}^{(\zeta)}}=0
$$

for $i=1,2, \cdots, t$. It is clear that the multiplicity label $\zeta=1,2, \cdots, d(n, t)$ in (37) is taken to be the label of different solutions $\left\{x^{(\zeta)}\right\}$ of Eq. (37). It can be verified that the number of solutions $d(n, t)$ of Eq. (37) equals exactly to the multiplicity in the reduction $j_{1} \otimes j_{2} \otimes \cdots \otimes$ $j_{n} \downarrow J$ with $j_{l}=\frac{1}{2}$ for $1 \leq l \leq n$, which can be calculated from Eq. (6) with

$$
\eta(n, t)=\sum_{\mu_{1}=0}^{1} \cdots \sum_{\mu_{n}=0}^{1} \delta_{q, t}
$$

for this case, in which $q=\sum_{i=1}^{n} \mu_{i}$. From Eqs. (6) and (38), the multiplicity $d(n, t)$ can be calculated recursively with $d(n, 0)=1$, which indicates that there are $d(n, t)$ different states with the same quasi-spin $S=n / 2-t$. For this case, there is a closed form of $d(n, t)$ with

$$
d(n, t)=\frac{(1+n-2 t) n !}{(1+n-t)(n-t) ! t !},
$$

which equals exactly to the dimension of the irrep $[n-t, t]$ of the permutation group $S_{n}[3,19]$ and is consistent with the result obtained from Eqs. (6) and (38). In this case, $c_{n, k}$ in the Van Vleck polynomials $(21)$ can be obtained in solving the corresponding Fuchsian equation (13) with

$$
c_{n, k}=-(n-k+1) k .
$$

Finally, the state with quasi-spin $S$ and any $M_{S}$ can be expressed as

$$
\left|\zeta ; S, M_{S}\right\rangle=\sqrt{\frac{\left(S+M_{S}\right) !}{(2 S) !\left(S-M_{S}\right) !}}\left(S^{-}\right)^{S-M_{S}}|\zeta ; S, S\rangle
$$

for the system with $p=n / 2+M_{S}$ pairs.

TABLE I: The multiplicity $d(8, t)$ for $S=4-t$ and $d(7, t)$ for $S=\frac{7}{2}-t$.

\begin{tabular}{ccc|cc}
\hline \hline$t$ & $S=4-t$ & $d(8, t)$ & $S=\frac{7}{2}-t$ & $d(7, t)$ \\
\hline 0 & 4 & 1 & $\frac{7}{2}$ & 1 \\
1 & 3 & 7 & $\frac{5}{2}$ & 6 \\
2 & 2 & 20 & $\frac{3}{2}$ & 14 \\
3 & 1 & 28 & $\frac{1}{2}$ & 14 \\
4 & 0 & 14 & & \\
\hline \hline
\end{tabular}

In order to demonstrate the method and properties of the zeros outlined in previous sections, in the following, we display results of the method for relatively simple cases with $n=8, t=1,4$ and $n=7, t=2,3$ as examples of even and odd $n$ case, respectively. The multiplicities $d(8, t)$ with $0 \leq t \leq 4$ and $d(7, t)$ with $0 \leq t \leq 3$ are listed in Table I. With parameters $\left\{\epsilon_{\alpha}\right\}$ chosen according to (29) and (30), we find there are exactly $d(n, t)$ different solutions for given $n$ and $t$ as shown in Table II-V. For any case, it can be verified that any zero $x_{i}^{(\zeta)}$ of $y_{t}^{(\zeta)}(x)$ indeed lies in one of the $n-1$ intervals $\left(\epsilon_{1}, \epsilon_{2}\right), \cdots,\left(\epsilon_{n-1}, \epsilon_{n}\right)$. It is obvious that $y_{1}^{(1)}(x)$ in Table II, $y_{4}^{(1)}(x), \cdots, y_{4}^{(6)}(x)$ in Table III, and $y_{2}^{(1)}(x), y_{2}^{(2)}(x)$ in Table IV are self-reflectional. While the solutions in most cases satisfy the reflection symmetry property (i). For example, $y_{4}^{(7)}(x)=y_{4}^{(8)}(-x), y_{4}^{(9)}(x)=y_{4}^{(10)}(-x)$, $y_{4}^{(11)}(x)=y_{4}^{(12)}(-x), y_{4}^{(13)}(x)=y_{4}^{(14)}(-x)$ when $n=8$ and $t=4$. The Van Vleck polynomial satisfies the same reflection property as that of the corresponding extended Heine-Stieltjes polynomial. In addition, one can verify that the Van Vleck zeros of $V_{n-2}^{(\zeta)}(x)$ indeed satisfy Eq. (20). With Stieltjes zeros $\left\{x_{i}\right\}$ of $y_{t}^{(\zeta)}(x)$ obtained from Table II-V, one can verify that the eigenstates (41) are mutually orthogonal with respect to the multiplicity label $\zeta$ :

$$
\left\langle\zeta ; S, M_{S} \mid \zeta^{\prime} ; S^{\prime}, M_{S}^{\prime}\right\rangle=\delta_{\zeta, \zeta^{\prime}} \delta_{S, S^{\prime}} \delta_{M_{S}, M_{S}^{\prime}} .
$$


TABLE II: The extended Heine-Stieltjes Polynomials $y_{1}^{(\zeta)}(x)$ for constructing $S=3$ states with $n=8$ and $t=1$ according to (35) and the corresponding Van Vleck Polynomials $V_{6}^{(\zeta)}(x)$.

\begin{tabular}{ccc}
\hline \hline \multicolumn{2}{c}{ The extended Heine-Stieltjes Polynomials $y_{1}^{(\zeta)}(x)$} & The Van Vleck Polynomials $V_{6}^{(\zeta)}(x)$ \\
\hline \hline$\zeta=1$ & $x$ & $-8(x-3.679)(x-2.59)(x-1.502)(x+1.502)(x+2.59)(x+3.679)$ \\
$\zeta=2$ & $x+3.679$ & $-8(x-3.679)(x-1.502)(x+1.502)(x+2.59)(x-2.59) x$ \\
$\zeta=3$ & $x-3.679$ & $-8(x+3.679)(x+1.502)(x-1.502)(x-2.59)(x+2.59) x$ \\
$\zeta=4$ & $x-2.59$ & $-8(x-3.679)(x+3.679)(x-1.502)(x+1.502)(x+2.59) x$ \\
$\zeta=5$ & $x+2.59$ & $-8(x+3.679)(x-3.679)(x+1.502)(x-1.502)(x-2.59) x$ \\
$\zeta=6$ & $x+1.502$ & $-8(x-3.679)(x-2.59)(x-1.502)(x+2.59)(x+3.679) x$ \\
$\zeta=7$ & $x-1.502$ & $-8(x+3.679)(x+2.59)(x+1.502)(x-2.59)(x-3.679) x$ \\
\hline \hline
\end{tabular}

Once the eigenstates (41) of (34) are obtained, the results can be used for constructing eigenstates and calculating eigenvalues of any mean-field plus pairing model by using the progressive diagonalization scheme as shown [19]. Furthermore, Eqs. (5) and (37) can be regarded as the same BAEs [20] in determining solutions of the mean-field plus the paring model in the strong pairing interaction $G \rightarrow \infty$ limit by replacing the parameters $\left\{\epsilon_{\alpha}\right\}$ with $\left\{2 \varepsilon_{\alpha}\right\}$, where $\left\{\varepsilon_{\alpha}\right\}$ are single-particle energies in the corresponding orbits of the mean-field [9].

\section{APPLICATION TO IDENTICAL-PARTICLE SYSTEMS}

Classification and construction of identical-particle states for a given angular momentum quantum number are fundamental, especially in nuclear structure theory. $n$-coupled states of $l$-bosons can be constructed as the basis vectors of symmetric irreducible representations of $U(2 l+1) \supset O(2 l+1) \supset O(3)$ as shown in [21-23], while those of $j$-fermions can be constructed as the basis vectors of antisymmetric irreducible representations of $U(2 j+1) \supset S p(2 j+1) \supset O(3)$ as shown in $[24,25]$. The Bethe ansatz method for angular momentum projection with the Heine-Stieltjes correspondence shown in previous sections can also be applied to construct states with definite angular momentum from a set of uncoupled single-particle product states for identicalparticle systems, which can be done as follows: Firstly, we solve the BAEs (5) for non-identical particle systems with the same spin, and then to construct the coupled state (2). Once the coupled state (2) is expanded in terms of single-particle product states, we take all particles to be identical, which is called assimilation. For identical-fermion systems, the Pauli principle forbidden single-particle product states will be automatically ruled out after the assimilation. Because of the additional permutation symmetry with respect to the single-particle coordinate permutations, the procedure outlined in previous sections can be simplified. In this section, we will show how the the procedure is taken.

\section{(1) Identical bosons}

Let the single-particle states of boson with angular momentum $l$ be $|l, m\rangle \equiv|m\rangle$ with $m=-l,-l+1, \cdots, l$. According to (2), $n$-coupled state with total angular momentum $L=n l-k$ and $M_{L}=L$

$$
\left.\left.\mid \zeta ; L, M_{L}=L\right)=L_{-}\left(x_{1}^{(\zeta)}\right) \cdots L_{-}\left(x_{k}^{(\zeta)}\right) \mid \text { h.w. }\right\rangle
$$

where $\mid$ h.w. $\rangle=\prod_{\alpha=1}^{n}\left|m_{\alpha}=l\right\rangle$ is the highest weight state,

$$
L_{-}\left(x_{i}^{(\zeta)}\right)=\sum_{\alpha=1}^{n} \frac{1}{x_{i}^{(\zeta)}-\epsilon_{\alpha}} L_{-}^{\alpha}
$$

in which the parameters $\left\{\epsilon_{\alpha}\right\}$ can usually be any set of unequal numbers, and $L_{-}^{\alpha}$ is the angular momentum lowering operator only acting on the $\alpha$-th copy of singleparticle state, and $L_{+}=\sum_{\alpha} L_{+}^{\alpha}$, similar to the nonidentical particle case. The corresponding BAEs is

$$
\sum_{\alpha=1}^{n} \frac{2 l}{x_{i}^{(\zeta)}-\epsilon_{\alpha}}-\sum_{t=1(\neq i)}^{k} \frac{2}{x_{i}^{(\zeta)}-x_{t}^{(\zeta)}}=0
$$


TABLE III: The extended Heine-Stieltjes Polynomials $y_{4}^{(\zeta)}(x)$ for constructing $S=0$ states with $n=8$ and $t=4$ according to (35) and the corresponding Van Vleck Polynomials $V_{6}^{(\zeta)}(x)$.

\begin{tabular}{|c|c|c|}
\hline & The extended Heine-Stieltjes Polynomials $y_{4}^{(\zeta)}(x)$ & The Van Vleck Polynomials $V_{6}^{(\zeta)}(x)$ \\
\hline$\zeta=1$ & $\left(x^{2}+0.379415\right)\left(x^{2}+10.53874\right)$ & $-20\left(x^{2}-13.0977\right)\left(x^{2}-6.2593\right)\left(x^{2}-1.9184\right)$ \\
\hline$\zeta=2$ & $\left(x^{2}-12.56879\right)\left(x^{2}+0.57829\right)$ & $-20\left(x^{2}-2.3151\right)\left(x^{2}-4.9901 x+6.4066\right)\left(x^{2}+4.9901 x+6.4066\right)$ \\
\hline$\zeta=3$ & $\left(x^{2}+0.8145\right)\left(x^{2}-5.7144\right)$ & $-20\left(x^{2}-12.8761\right)\left(x^{2}-2.8274 x+2.1700\right)\left(x^{2}+2.8274 x+2.1700\right)$ \\
\hline$\zeta=4$ & $\left(x^{2}-2.23204\right)\left(x^{2}-12.3149\right)$ & $-20\left(x^{2}+0.5558\right)\left(x^{2}-5.3854 x+7.4056\right)\left(x^{2}+5.3854 x+7.4056\right)$ \\
\hline$\zeta=5$ & $\left(x^{2}+4.82433\right)\left(x^{2}-1.80406\right)$ & $-20\left(x^{2}-13.0201\right)\left(x^{2}-6.1361\right)\left(x^{2}+0.2502\right)$ \\
\hline$\zeta=6$ & $\left(x^{2}-5.07233 x+6.61367\right)\left(x^{2}+5.07233 x+6.61367\right)$ & $-20\left(x^{2}-12.5294\right)\left(x^{2}-2.2932\right)\left(x^{2}+0.5729\right)$ \\
\hline$\zeta=7$ & $\left(x^{2}-1.2051 x-8.3519\right)\left(x^{2}+1.2051 x+0.8179\right)$ & $\begin{array}{c}-20\left(x^{2}+2.0270 x-5.5609\right)\left(x^{2}-4.8900 x+6.1511\right) \times \\
\left(x^{2}+2.8630 x+2.2150\right)\end{array}$ \\
\hline$\zeta=8$ & $\left(x^{2}+1.2051 x-8.3519\right)\left(x^{2}-1.2051 x+0.8179\right)$ & $\begin{array}{c}-20\left(x^{2}-2.0270 x-5.5609\right)\left(x^{2}+4.8900 x+6.1511\right) \times \\
\left(x^{2}-2.8630 x+2.2150\right)\end{array}$ \\
\hline$\zeta=9$ & $\left(x^{2}-5.01525 x+6.47201\right)\left(x^{2}+5.01525 x+5.28038\right)$ & $\begin{array}{c}-20\left(x^{2}+5.0460 x+5.3283\right)\left(x^{2}+0.3380 x+0.5727\right) \times \\
\left(x^{2}-5.3840 x+7.4014\right)\end{array}$ \\
\hline$\zeta=10$ & $\left(x^{2}+5.01525 x+6.47201\right)\left(x^{2}-5.01525 x+5.28038\right)$ & $\begin{array}{c}-20\left(x^{2}-5.0460 x+5.3283\right)\left(x^{2}-0.3380 x+0.5727\right) \times \\
\left(x^{2}+5.3840 x+7.4014\right)\end{array}$ \\
\hline$\zeta=11$ & $\left(x^{2}+2.162707 x+3.56055\right)\left(x^{2}-2.162707 x-5.00227\right)$ & $\begin{array}{c}-20\left(x^{2}+6.051 x+8.8268\right)\left(x^{2}-1.3451 x-0.3943\right) \times \\
\left(x^{2}-4.7057 x+5.6852\right)\end{array}$ \\
\hline$\zeta=12$ & $\left(x^{2}-2.162707 x+3.56055\right)\left(x^{2}+2.162707 x-5.00227\right)$ & $\begin{array}{c}-20\left(x^{2}-6.051 x+8.8268\right)\left(x^{2}+1.3451 x-0.3943\right) \times \\
\left(x^{2}+4.7057 x+5.6852\right)\end{array}$ \\
\hline$\zeta=13$ & $\left(x^{2}+1.0751 x+2.9229\right)\left(x^{2}-1.0751 x-3.2612\right)$ & $\begin{array}{c}-20\left(x^{2}-3.3149 x-1.0537\right)\left(x^{2}-2.7178 x+2.1494\right) \times \\
\left(x^{2}+6.0327 x+8.7374\right)\end{array}$ \\
\hline$\zeta=14$ & $\left(x^{2}-1.0751 x+2.9229\right)\left(x^{2}+1.0751 x-3.2612\right)$ & $\begin{array}{c}-20\left(x^{2}+3.3149 x-1.0537\right)\left(x^{2}+2.7178 x+2.1494\right) \times \\
\left(x^{2}-6.0327 x+8.7374\right)\end{array}$ \\
\hline
\end{tabular}

for $i=1,2, \cdots, k$. By substituting the solutions $\left\{x_{i}\right\}$ of (45) into (43), (43) gives final result after assimilation.

It can be easily proven that the $n$-coupled state with $L=l n-1$ is zero. Because

$$
\begin{gathered}
\left.L_{-}^{\alpha} \mid \text { h.w. }\right\rangle=\sqrt{2 l} \prod_{\beta=1(\neq \alpha)}^{n}\left|m_{\beta}=l\right\rangle\left|m_{\alpha}=l-1\right\rangle \\
=\sqrt{2 l} \prod_{\beta=1}^{n-1}\left|m_{\beta}=l\right\rangle\left|m_{n}=l-1\right\rangle
\end{gathered}
$$

due to the fact that these bosons are identical, (43) becomes

$$
\begin{gathered}
\left.\left.\mid \zeta ; L=M_{L}=n l-1\right)=L_{-}\left(x^{(\zeta)}\right) \mid \text { h.w. }\right\rangle= \\
\sqrt{2 l} \sum_{\alpha=1}^{n} \frac{2 l}{x^{(\zeta)}-\epsilon_{\alpha}} \prod_{\beta=1}^{n-1}\left|m_{\beta}=l\right\rangle\left|m_{n}=l-1\right\rangle
\end{gathered}
$$

which is zero because 
TABLE IV: The extended Heine-Stieltjes Polynomials $y_{2}^{(\zeta)}(x)$ for constructing $S=3 / 2$ states with $n=7$ and $t=2$ according to (35) and the corresponding Van Vleck Polynomials $V_{5}^{(\zeta)}(x)$.

\begin{tabular}{|c|c|c|}
\hline & The extended Heine-Stieltjes Polynomials $y_{2}^{(\zeta)}(x)$ & The Van Vleck Polynomials $V_{5}^{(\zeta)}(x)$ \\
\hline$\zeta=1$ & $(x-2.646)(x+2.646)$ & $-12(x-1.5275)(x+1.5275) x^{3}$ \\
\hline$\zeta=2$ & $(x-1.5275)(x+1.5275)$ & $-12(x-2.646)(x+2.646) x^{3}$ \\
\hline$\zeta=3$ & $x^{2}-1.270 x+0.5564$ & $-12(x-2.63447)(x-1.4683 x)(x+0.430964)(x+1.54863)(x+2.65236 x)$ \\
\hline$\zeta=4$ & $x^{2}+1.270 x+0.5564$ & $-12(x+2.63447)(x+1.4683 x)(x-0.430964)(x-1.54863)(x-2.65236 x)$ \\
\hline$\zeta=5$ & $(x-2.582)(x-0.6325)$ & $-12(x+0.4792)(x+1.5636)(x+2.6585)\left(x^{2}-3.3621 x+2.9644\right)$ \\
\hline$\zeta=6$ & $(x+2.582)(x+0.6325)$ & $-12(x-0.4792)(x-1.5636)(x-2.6585)\left(x^{2}+3.3621 x+2.9644\right)$ \\
\hline$\zeta=7$ & $(x-2.620)(x+0.4611)$ & $-12(x-0.779279)(x+1.5569)(x+2.6556)\left(x^{2}-2.5336 x+1.66393\right)$ \\
\hline$\zeta=8$ & $(x+2.620)(x-0.4611)$ & $-12(x+0.779279)(x-1.5569)(x-2.6556)\left(x^{2}+2.5336 x+1.66393\right)$ \\
\hline$\zeta=9$ & $(x-1.484)(x+0.4196)$ & $-12(x-2.63681)(x+1.5462)(x+2.6515)\left(x^{2}-1.1171 x+0.4745\right)$ \\
\hline$\zeta=10$ & $(x+1.484)(x-0.4196)$ & $-12(x+2.63681)(x-1.5462)(x-2.6515)\left(x^{2}+1.1171 x+0.4745\right)$ \\
\hline$\zeta=11$ & $x^{2}-3.831 x+3.719$ & $-12(x-2.5078)(x-0.6085)(x+0.4859)(x+1.5666)(x+2.6598)$ \\
\hline$\zeta=12$ & $x^{2}+3.831 x+3.719$ & $-12(x+2.5078)(x+0.6085)(x-0.4859)(x-1.5666)(x-2.6598)$ \\
\hline$\zeta=13$ & $(x-2.637)(x+1.546)$ & $-12(x-1.4827)(x+0.4211)(x+2.6516)\left(x^{2}-1.1358 x+0.4844\right)$ \\
\hline$\zeta=14$ & $(x+2.637)(x-1.546)$ & $-12(x+1.4827)(x-0.4211)(x-2.6516)\left(x^{2}+1.1358 x+0.4844\right)$ \\
\hline
\end{tabular}

$$
\sum_{\alpha=1}^{n} \frac{2 l}{x^{(\zeta)}-\epsilon_{\alpha}}=0
$$

according to Eq. (45) when $k=1$.

When $k \geq 2$, the number of states (43) with $L=2 l-k$ may be calculated in the following way: Let $P_{n}(k)$ be number of different $n$-partitions of the integer $k$ with $k=\sum_{i=1}^{n} \xi_{i}$, where $2 l \geq \xi_{1} \geq \xi_{2} \geq \cdots \geq \xi_{n} \geq 0$. Then, the number of linearly independent states shown in (43) for $l$-bosons $D_{\mathrm{B}}(n, k)=P_{n}(k)-P_{n}(k-1)$, which gives the multiplicity of given $L=n l-k$ in the reduction $U(2 l+1) \downarrow O(3)$ for the symmetric irreducible representation $[n, \dot{0}]$ of $U(2 l+1)$. Generally, $D_{\mathrm{B}}(n, k)$ is far less than $d(n, k)$ shown in (6) for non-identical particles. Therefore, for given $L, n$-coupled states (43) obtained from solutions of (45) are over-complete for identicalparticle systems when $k \geq 2$. Actually, (43) obtained from different solutions of (45), up to a normalization constant, are all the same when $D_{\mathrm{B}}(n, k)=1$. When
$D_{\mathrm{B}}(n, k) \geq 2$, the solutions (43) are not orthogonal with respect to the multiplicity label, and many solutions of (43) can be expressed by a linear combination of other solutions of (43).

Simplification can be made to overcome such complexity mainly because there is a freedom to choose the parameters $\left\{\epsilon_{\alpha}\right\}$ in $(44)$. When $D_{\mathrm{B}}(n, k)=1$, we set

$$
\left\{\begin{array}{c}
\epsilon_{\alpha}=-1 \text { for } \alpha \leq p \\
\epsilon_{\alpha+p}=1 \text { for } \alpha \geq 1
\end{array}\right.
$$

when $n=2 p$, and

$$
\left\{\begin{array}{l}
\epsilon_{\alpha}=-1 \alpha \leq p+1 \\
\epsilon_{\alpha+p+1}=1 \text { for } \alpha \geq 1
\end{array}\right.
$$

when $n=2 p+1$. With such choice, Eq. (45) becomes 
TABLE V: The extended Heine-Stieltjes Polynomials $y_{3}^{(\zeta)}(x)$ for constructing $S=1 / 2$ states with $n=7$ and $t=3$ according to (35) and the corresponding Van Vleck Polynomials $V_{5}^{(\zeta)}(x)$.

\begin{tabular}{|c|c|c|}
\hline & The extended Heine-Stieltjes Polynomials $y_{3}^{(\zeta)}(x)$ & The Van Vleck Polynomials $V_{5}^{(\zeta)}(x)$ \\
\hline$\zeta=1$ & $(x-0.6354)\left(x^{2}-2.020 x+2.296\right)$ & $-15(x-2.581)(x-1.432)(x+0.4002)(x+1.514)(x+2.630)$ \\
\hline$\zeta=2$ & $(x+0.6354)\left(x^{2}+2.020 x+2.296\right)$ & $-15(x+2.581)(x+1.432)(x-0.4002)(x-1.514)(x-2.630)$ \\
\hline$\zeta=3$ & $(x-2.542)\left(x^{2}+0.5857 x+0.2296\right)$ & $-15(x-0.4874)(x+1.473)(x+2.619)\left(x^{2}-3.213 x+2.754\right)$ \\
\hline$\zeta=4$ & $(x+2.542)\left(x^{2}-0.5857 x+0.2296\right)$ & $-15(x+0.4874)(x-1.473)(x-2.619)\left(x^{2}+3.213 x+2.754\right)$ \\
\hline$\zeta=5$ & $(x-2.582)(x+0.5705)(x+2.540)$ & $-15(x-0.6329)\left(x^{2}-2.867 x+2.204\right)\left(x^{2}+3.394 x+3.026\right)$ \\
\hline$\zeta=6$ & $(x+2.582)(x-0.5705)(x-2.540)$ & $-15(x+0.6329)\left(x^{2}+2.867 x+2.204\right)\left(x^{2}-3.394 x+3.026\right)$ \\
\hline$\zeta=7$ & $(x-0.3106)\left(x^{2}+2.634 x+1.912\right)$ & $-15(x-2.625)(x-1.498)(x+2.562)\left(x^{2}+1.097 x+0.4387\right)$ \\
\hline$\zeta=8$ & $(x+0.3106)\left(x^{2}-2.634 x+1.912\right)$ & $-15(x+2.625)(x+1.498)(x-2.562)\left(x^{2}-1.097 x+0.4387\right)$ \\
\hline$\zeta=9$ & $(x-1.434)(x+0.5286)(x+2.529)$ & $-15(x-2.612)\left(x^{2}-1.116 x+0.4798\right)\left(x^{2}+3.403 x+3.044\right)$ \\
\hline$\zeta=10$ & $(x+1.434)(x-0.5286)(x-2.529)$ & $-15(x+2.612)\left(x^{2}+1.116 x+0.4798\right)\left(x^{2}-3.403 x+3.044\right)$ \\
\hline$\zeta=11$ & $(x-2.593)\left(x^{2}+3.468 x+3.144\right)$ & $-15(x-0.7007)(x+0.5593)(x+2.528)\left(x^{2}-2.562 x+1.738\right)$ \\
\hline$\zeta=12$ & $(x+2.593)\left(x^{2}-3.468 x+3.144\right)$ & $-15(x+0.7007)(x-0.5593)(x-2.528)\left(x^{2}+2.562 x+1.738\right)$ \\
\hline$\zeta=13$ & $(x-1.461)\left(x^{2}+3.299 x+2.884\right)$ & $-15(x-2.616)(x+0.5086)(x+2.537)\left(x^{2}-0.7961 x+0.3266\right)$ \\
\hline$\zeta=14$ & $(x+1.461)\left(x^{2}-3.299 x+2.884\right)$ & $-15(x+2.616)(x-0.5086)(x-2.537)\left(x^{2}+0.7961 x+0.3266\right)$ \\
\hline
\end{tabular}

$$
\frac{2 l(p+r)}{x_{i}+1}+\frac{2 l p}{x_{i}-1}-\sum_{t=1(\neq i)}^{k} \frac{2}{x_{i}-x_{t}}=0
$$

for $i=1,2, \cdots, k$, where $r=0$ when $n=2 p$ and $r=1$ when $n=2 p+1$, which are exactly the Niven equations for zeros of the Jacobi polynomial $P_{k}^{[-2 l p-1,-2 l(p+r)-1]}(x)$. There is only one set of zeros of (51) which is sufficient for (43) when $D_{\mathrm{B}}(n, k)=$ 1. Therefore, (43) with zeros of the Jacobi polynomial $P_{k}^{[-2 l p-1,-2 l(p+r)-1]}(x)$ are $n$-coupled states with $L=n l-k$ when the parameters $\left\{\epsilon_{\alpha}\right\}$ are chosen according to $(49)$ or $(50)$ when $D_{\mathrm{B}}(n, k)=1$.

For example, there is only one state with $L=6$ for $n=4 d$-bosons $(l=2)$. According to (49), we set $\left\{\epsilon_{1}=\epsilon_{2}=-1, \epsilon_{3}=\epsilon_{4}=1\right\}$. Substituting two zeros $\left\{x_{1}=-0.2582 \imath, x_{2}=0.2582 \imath\right\}$ of the Jacobi polynomial $P_{2}^{[-7,-7]}(x)$ into $(43)$, we get

$$
\left|L=M_{L}=6\right\rangle=-0.5222|2,2,1,1\rangle+0.8528|2,2,2,0\rangle
$$

after assimilation and normalization.

When $D_{\mathrm{B}}(n, k) \geq 2$, we have many ways to set the parameters $\left\{\epsilon_{\alpha}\right\}$. The simplest way is to choose the twovalue parameterization with $\epsilon_{\alpha_{1}}=\epsilon_{\alpha_{2}}=\cdots=\epsilon_{\alpha_{r}}=-1$ and the rest parameters $\epsilon_{\beta}=1$ when $\beta \neq \alpha_{i}$ for $i=1,2, \cdots, r$. Obviously, there are $2^{n}-2$ different ways of such parameterization, from which one can choose $D_{\mathrm{B}}(n, k)$ of them. Zeros of the corresponding Jacobi polynomial can be used to obtain the final results from (43). It seems that $D_{\mathrm{B}}(n, k) \leq 2^{n}-2$ is always satisfied for $n \geq 2$ though we are unable to prove this inequality in general. Therefore, the above two-value parameterization seems sufficient to resolve the multiplicity.

For example, there are two coupled states of $n=4$ $d$-bosons with $L=4$. One can set $\left\{\epsilon_{1}=\epsilon_{2}=-1, \epsilon_{3}=\right.$ $\left.\epsilon_{4}=1\right\}$ for one solution with 


$$
\begin{gathered}
\left|\zeta=1, L=M_{L}=4\right\rangle=0.2208|1,1,1,1\rangle- \\
0.7211|2,1,1,0\rangle+0.6403|2,2,0,0\rangle- \\
0.1030|2,2,1,-1\rangle+0.1030|2,2,2,-2\rangle
\end{gathered}
$$

and set $\left\{\epsilon_{1}=-1, \epsilon_{2}=\epsilon_{3}=\epsilon_{4}=1\right\}$ for another solution with

$$
\begin{gathered}
\left|\zeta=2, L=M_{L}=4\right\rangle=0.0827|1,1,1,1\rangle- \\
0.2702|2,1,1,0\rangle-0.1161|2,2,0,0\rangle+ \\
0.6733|2,2,1,-1\rangle-0.6733|2,2,2,-2\rangle .
\end{gathered}
$$

In this case, the final coupled states (53) and (54) are not orthogonal with respect to the multiplicity label $\zeta$, namely $\langle\zeta=1 \mid \zeta=2\rangle \neq 0$. In order to be orthonormalized, the Gram-Schimidt process may be adopted.

More complicated parameterizations are always possible. For example, we can also set

$$
\left\{\begin{array}{l}
\epsilon_{\alpha}=-1 \text { for } \alpha \leq p, \\
\epsilon_{p+1}=0, \\
\epsilon_{\beta+p+1}=1 \text { for } \beta \geq 1,
\end{array}\right.
$$

where the integer $p$ can arbitrarily be chosen. Thus, the BAEs (45) become

$\frac{2 l p}{x_{i}^{(\zeta)}+1}+\frac{2 l}{x_{i}^{(\zeta)}}+\frac{2 l(n-p-1)}{x_{i}^{(\zeta)}-1}-\sum_{t=1(\neq i)}^{k} \frac{2}{x_{i}^{(\zeta)}-x_{t}^{(\zeta)}}=0$

for $i=1,2, \cdots, k$. Then, one can choose $D_{\mathrm{B}}(n, k)$ solutions of $(56)$ to get the results. When we set $\left\{\epsilon_{1}=\right.$ $\left.-1, \epsilon_{2}=0, \epsilon_{3}=\epsilon_{4}=1\right\}$ for the previous $L=4$ example of $4 d$-bosons, there are 5 solutions of (56) with the extended Heine-Stieltjes polynomials shown in Table VI.

\begin{tabular}{|c|c|}
\hline & The extended Heine-Stieltjes Polynomials $y_{4}^{(\zeta)}(x)$ \\
\hline$\zeta=1$ & $\left(0.1382-0.7053 x+x^{2}\right)\left(0.2800-0.6172 x+x^{2}\right)$ \\
\hline$\zeta=2$ & $\left(0.6106+1.5493 x+x^{2}\right)\left(0.9545+1.8343 x+x^{2}\right)$ \\
\hline$\zeta=3$ & $(-0.2918+x)(0.4284+x)\left(0.1284-0.4857 x+x^{2}\right)$ \\
\hline$\zeta=4$ & $\left(0.0876-0.5298 x+x^{2}\right)\left(0.3138+1.0483 x+x^{2}\right)$ \\
\hline$\zeta=5$ & $(-0.2874+x)(0.6446+x)\left(0.6007+1.4124 x+x^{2}\right)$ \\
\hline
\end{tabular}

The corresponding coupled states after normalization are

$$
\begin{gathered}
\left|\zeta=1, L=M_{L}=4\right\rangle=0.1974|1,1,1,1\rangle- \\
0.6448|2,1,1,0\rangle+0.6502|2,2,0,0\rangle- \\
0.2475|2,2,1,-1\rangle+0.2475|2,2,2,-2\rangle ; \\
\left|\zeta=2, L=M_{L}=4\right\rangle=-0.0432|1,1,1,1\rangle+
\end{gathered}
$$

TABLE VI: The extended Heine-Stieltjes Polynomials $y_{4}^{(\zeta)}(x)$ for $n=4 d$-bosons coupled to $L=M_{L}=4$ with $\left\{\epsilon_{1}=\right.$ $\left.-1, \epsilon_{2}=0, \epsilon_{3}=\epsilon_{4}=1\right\}$.

$$
\begin{gathered}
0.1412|2,1,1,0\rangle+0.2252|2,2,0,0\rangle- \\
0.6810|2,2,1,-1\rangle+0.6810|2,2,2,-2\rangle ;
\end{gathered}
$$$$
\left|\zeta=3, L=M_{L}=4\right\rangle=0.1926|1,1,1,1\rangle-
$$$$
0.6290|2,1,1,0\rangle+0.2642|2,2,0,0\rangle+
$$$$
0.4987|2,2,1,-1\rangle-0.4987|2,2,2,-2\rangle \text {; }
$$$$
\left|\zeta=4, L=M_{L}=4\right\rangle=0.0829|1,1,1,1\rangle-
$$$$
0.2707|2,1,1,0\rangle+0.5085|2,2,0,0\rangle-
$$$$
0.5750|2,2,1,-1\rangle+0.5750|2,2,2,-2\rangle \text {; }
$$$$
\left|\zeta=5, L=M_{L}=4\right\rangle=-0.2355|1,1,1,1\rangle+
$$$$
0.7692|2,1,1,0\rangle-0.5740|2,2,0,0\rangle-
$$$$
0.1081|2,2,1,-1\rangle+0.1081|2,2,2,-2\rangle .
$$

Because $D_{\mathrm{B}}(4,4)=2$ in this case, we may choose

$$
|\chi=1\rangle=|\zeta=1\rangle
$$

$$
|\chi=2\rangle=c_{1}|\zeta=1\rangle+c_{2}|\zeta=2\rangle
$$


where $c_{1}=1 / \mathcal{N}$ and $c_{2}=-\frac{1}{\mathcal{N}\langle\zeta=1 \mid \zeta=2\rangle}$ with the normalization constant

$$
\mathcal{N}=\left(\langle\zeta=1 \mid \zeta=1\rangle+\frac{\langle\zeta=2 \mid \zeta=2\rangle}{\langle\zeta=1 \mid \zeta=2\rangle^{2}}-2\right)^{1 / 2}
$$

according to the Gram-Schmidt process. Then, one finds

$$
\begin{gathered}
|\zeta=3\rangle=0.3686|\chi=1\rangle+0.9296|\chi=2\rangle, \\
|\zeta=4\rangle=0.8062|\chi=1\rangle-0.59168|\chi=2\rangle, \\
|\zeta=5\rangle=-0.8622|\chi=1\rangle-0.5066|\chi=2\rangle .
\end{gathered}
$$

This example shows that coupled states with zeros of other polynomials can indeed be expressed as linear combinations of the chosen two due to the overcompleteness.

\section{(2) Identical fermions}

Let the single-particle states of fermions with spin $j$ be $|j, m\rangle \equiv|m\rangle$ with $m=-j,-j+1, \cdots, j$. Unlike identical bosons, we have verified that the parameters $\left\{\epsilon_{\alpha}\right\}$ must be a set of unequal numbers for identical fermions. Initially, we need to solve BAEs (5) for non-identical particles with the same spin $j_{\alpha}=j$ for $\alpha=1,2, \cdots, n$. After (2) is expanded in terms of the single-particle product states, we then take all particles to be identical. The Pauli exclusion will automatically rule out any forbidden single-particle product states after such assimilation. The result of (2) gives final coupled state with total angular momentum $J=n j-k$ and $M_{J}=J$. However, the two-value parameterization schemes for identical bosons shown previously can not be used for identical fermions mainly because the single-particle product states are totally antisymmetric with respect to permutations among different single-particle states. As a consequence, the coupled state is zero if one choose any two-value parameterization scheme in $\left\{\epsilon_{\alpha}\right\}$ for identical fermions.

Similar to identical bosons, the number of linearly independent states obtained from $(2), D_{\mathrm{F}}(n, k)$, can be calculated as follows: Let $Q_{n}(k)$ be number of different $n$-partitions of the integer $k$ with $k=\sum_{i=1}^{n} \xi_{i}$, where $2 j+1-n \geq \xi_{1} \geq \xi_{2} \geq \cdots \geq \xi_{n} \geq 0$. Then, the number of linearly independent states obtained from (2) for $j$-fermions, $D_{\mathrm{F}}(n, k)=Q_{n}(k)-Q_{n}(k-1)$, which gives the multiplicity of given $J=n j-k$ in the reduction $U(2 j+1) \downarrow O(3)$ for the antisymmetric irreducible representation $\left[1^{n}, \dot{0}\right]$ of $U(2 l+1)$. Generally, $D_{\mathrm{F}}(n, k)$ is far less than $d(n, k)$ shown in (6) for non-identical particles. Therefore, (2) obtained from solutions of (5) are also over-complete. Similar to identical bosons, one only needs to choose $D_{\mathrm{F}}(n, k)$ solutions of $(5)$. When the parameters $\left\{\epsilon_{\alpha}\right\}$ are chosen according to (29) or (30), the coupled state (2) satisfies the symmetry

$$
\begin{gathered}
\left.J_{-}\left(x_{1}^{(\zeta)}\right) J_{-}\left(x_{2}^{(\zeta)}\right) \cdots J_{-}\left(x_{k}^{(\zeta)}\right) \mid \text { h.w. }\right\rangle \\
\left.=J_{-}\left(-x_{1}^{(\zeta)}\right) J_{-}\left(-x_{2}^{(\zeta)}\right) \cdots J_{-}\left(-x_{k}^{(\zeta)}\right) \mid \text { h.w. }\right\rangle
\end{gathered}
$$

Therefore, only one of reflectional symmetry pair of Stieltjes zeros should be considered.

In the following, we take $n=3 j=9 / 2$ identical fermions as examples. In this case, there is only one coupled state with $J=M_{J}=17 / 2(k=5)$, for which there are 6 extended Heine-Stieltjes polynomials as shown in Table VII, which clearly shows that $y_{5}^{(2)}(x)=y_{5}^{(1)}(-x)$, $y_{5}^{(4)}(x)=y_{5}^{(3)}(-x)$, and $y_{5}^{(6)}(x)=y_{5}^{(5)}(-x)$. But three solutions $y_{5}^{(1)}(x), y_{5}^{(3)}(x)$, and $y_{5}^{(5)}(x)$ all result in one coupled state

$$
\left|J=M_{J}=17 / 2\right\rangle=0.7746|9 / 2,5 / 2,3 / 2\rangle-
$$

$0.6325|9 / 2,7 / 2,1 / 2\rangle$

up to a normalization constant after assimilation.

TABLE VII: The extended Heine-Stieltjes Polynomials $y_{5}^{(\zeta)}(x)$ for $J=M_{J}=17 / 2(k=5)$ coupled states of $n=3$ $j=9 / 2$ identical fermions with $\left\{\epsilon_{1}=-1, \epsilon_{2}=0, \epsilon_{3}=1\right\}$.

The extended Heine-Stieltjes Polynomials $y_{5}^{(\zeta)}(x)$

$$
\begin{array}{cc}
\zeta=1 & (-0.6191+x)\left(0.60249-1.3616 x+x^{2}\right) \times \\
& \left(0.4249-1.2622 x+x^{2}\right) \\
\zeta=2 & (0.6191+x)\left(0.60249+1.3616 x+x^{2}\right) \times \\
& \left(0.4249+1.2622 x+x^{2}\right) \\
\zeta=3 & (0.4849+x)\left(0.4559-1.2046 x+x^{2}\right) \times \\
& \left(0.34201-1.1556 x+x^{2}\right) \\
\zeta=4 & (-0.4849+x)\left(0.4559+1.2046 x+x^{2}\right) \times \\
& \left(0.34201+1.1556 x+x^{2}\right) \\
\zeta=5 & (0.5371+x)\left(0.2723-1.0146 x+x^{2}\right) \times \\
& (-0.5371+x)\left(0.2723+1.0146 x+x^{2}\right) \times \\
\zeta=6 & \left(0.3451+1.0889 x+x^{2}\right) \\
\hline \hline
\end{array}
$$

Since $D_{\mathrm{F}}(3,9)=2$ for $j=9 / 2$ identical fermions, $J=9 / 2$ should occur twice. While there are $10 \mathrm{ex}-$ tended Heine-Stieltjes polynomials as shown in Table 
TABLE VIII: The extended Heine-Stieltjes Polynomials $y_{9}^{(\zeta)}(x)$ for $J=9 / 2$ coupled states of $n=3 j=9 / 2$ identical fermions with $\left\{\epsilon_{1}=-1, \epsilon_{2}=0, \epsilon_{3}=1\right\}$.

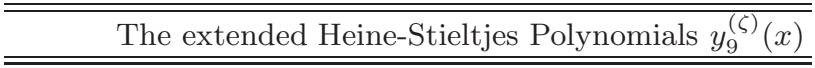

$$
\begin{aligned}
& \zeta=1\left(1.7186-2.3241 x+x^{2}\right)\left(0.9532-1.7796 x+x^{2}\right) \times \\
& \left(0.6707-1.5615 x+x^{2}\right)\left(0.5516-1.4664 x+x^{2}\right) \times \\
& (-0.7196+x) \\
& \zeta=2\left(1.7186+2.3241 x+x^{2}\right)\left(0.9532+1.7796 x+x^{2}\right) \times \\
& \left(0.6707+1.5615 x+x^{2}\right)\left(0.5516+1.4664 x+x^{2}\right) \times \\
& (0.7196+x) \\
& \zeta=3\left(1.3423-1.8543 x+x^{2}\right)\left(0.7134-1.4807 x+x^{2}\right) \times \\
& \left(0.4992-1.3433 x+x^{2}\right)\left(0.4228-1.2929 x+x^{2}\right) \times \\
& (0.4142+x) \\
& \zeta=4\left(1.3423+1.8543 x+x^{2}\right)\left(0.7134+1.4807 x+x^{2}\right) \times \\
& \left(0.4992+1.3433 x+x^{2}\right)\left(0.4228+1.2929 x+x^{2}\right) \times \\
& (-0.4142+x) \\
& \zeta=5\left(0.9728-1.4201 x+x^{2}\right)\left(0.5034-1.2196 x+x^{2}\right) \times \\
& \left(0.3611-1.1571 x+x^{2}\right)\left(0.1818+0.8182 x+x^{2}\right) \times \\
& (-0.5708+x) \\
& \zeta=6\left(0.9728+1.4201 x+x^{2}\right)\left(0.5034+1.2196 x+x^{2}\right) \times \\
& \left(0.3611+1.1571 x+x^{2}\right)\left(0.1818-0.8182 x+x^{2}\right) \times \\
& (0.5708+x) \\
& \zeta=7\left(0.6395-1.0463 x+x^{2}\right)\left(0.3362-1.0087 x+x^{2}\right) \times \\
& \left(0.2589-1.0027 x+x^{2}\right)\left(0.2069+0.7932 x+x^{2}\right) \times \\
& (0.4142+x) \\
& \zeta=8\left(0.6395+1.0463 x+x^{2}\right)\left(0.3362+1.0087 x+x^{2}\right) \times \\
& \left(0.2589+1.0027 x+x^{2}\right)\left(0.2069-0.7932 x+x^{2}\right) \times \\
& (-0.4142+x) \\
& \zeta=9\left(0.1801-0.8267 x+x^{2}\right)\left(0.2527-0.7578 x+x^{2}\right) \times \\
& \left(0.3775+0.7973 x+x^{2}\right)\left(0.2253+0.8689 x+x^{2}\right) \times \\
& (0.4423+x) \\
& \zeta=10\left(0.1801+0.8267 x+x^{2}\right)\left(0.2527+0.7578 x+x^{2}\right) \times \\
& \left(0.3775-0.7973 x+x^{2}\right)\left(0.2253-0.8689 x+x^{2}\right) \times \\
& (-0.4423+x)
\end{aligned}
$$

VIII, which shows that $y_{9}^{(2)}(x)=y_{9}^{(1)}(-x), y_{9}^{(4)}(x)=$ $y_{9}^{(3)}(-x), y_{9}^{(6)}(x)=y_{9}^{(5)}(-x), y_{9}^{(8)}(x)=y_{9}^{(7)}(-x)$, and $y_{9}^{(10)}(x)=y_{9}^{(9)}(-x)$, we only need to choose 2 of them to get the coupled states according to (2). The coupled state with $y_{9}^{(1)}(x)$ is

$$
\left|\zeta=1, J=M_{J}=9 / 2\right\rangle=0.2105|5 / 2,3 / 2,1 / 2\rangle-
$$

$0.1684|7 / 2,3 / 2,-1 / 2\rangle+0.1575|7 / 2,5 / 2,-3 / 2\rangle-$

$0.3384|9 / 2,1 / 2,-1 / 2\rangle+0.4415|9 / 2,3 / 2,-3 / 2\rangle-$

$$
0.5446|9 / 2,5 / 2,-5 / 2\rangle+0.5446|9 / 2,7 / 2,-7 / 2\rangle,
$$

and that with $y_{9}^{(3)}(x)$ is

$$
\left|\zeta=2, J=M_{J}=9 / 2\right\rangle=0.1506|5 / 2,3 / 2,1 / 2\rangle-
$$

$0.1205|7 / 2,3 / 2,-1 / 2\rangle+0.1127|7 / 2,5 / 2,-3 / 2\rangle-$

$0.3913|9 / 2,1 / 2,-1 / 2\rangle+0.4651|9 / 2,3 / 2,-3 / 2\rangle-$

$0.5388|9 / 2,5 / 2,-5 / 2\rangle+0.5388|9 / 2,7 / 2,-7 / 2\rangle$.

After the Gram-Schmidt orthonormalization, we have

$$
\begin{aligned}
& \left|\chi=1, J=M_{J}=9 / 2\right\rangle=|\zeta=1, J=M=9 / 2\rangle, \\
& \left|\chi=2, J=M_{J}=9 / 2\right\rangle=0.5526|5 / 2,3 / 2,1 / 2\rangle- \\
& 0.4421|7 / 2,3 / 2,-1 / 2\rangle+0.4135|7 / 2,5 / 2,-3 / 2\rangle+ \\
& 0.5164|9 / 2,1 / 2,-1 / 2\rangle-0.2457|9 / 2,3 / 2,-3 / 2\rangle- \\
& 0.0250|9 / 2,5 / 2,-5 / 2\rangle+0.0250|9 / 2,7 / 2,-7 / 2\rangle .
\end{aligned}
$$

Then, other 3 coupled states corresponding to $y_{9}^{(5)}(x)$, $y_{9}^{(7)}(x), y_{9}^{(9)}(x)$, respectively, can be expressed as linear combinations of $|\chi=1\rangle$ and $|\chi=2\rangle$.

\section{SUMMARY}

In summary, a new angular momentum projection for many-particle systems is formulated based on the HeineStieltjes correspondence, which can be regarded as the solutions of the mean-field plus the paring model in the strong pairing interaction $G \rightarrow \infty$ limit [9]. With the special choice of the parameters $\left\{\epsilon_{\alpha}\right\}$, the solutions of the associated BAEs are simplified because of the additional reflection symmetries. Properties of the Stieltjes zeros and the related Van Vleck zeros are discussed. The electrostatic interpretation of these zeros are presented. As an example, the application to $n$ nonidentical particles with spin- $1 / 2$ is made to elucidate the procedure and properties of the Stieltjes zeros and the related Van Vleck 
zeros. It is clear that the new angular momentum projection can be used for nonidentical-particles with arbitrary spins. It is shown that the new angular momentum projection for identical bosons or fermions can be simplified with the branching multiplicity formula of $U(N) \downarrow O(3)$ and the special choices of the parameters used in the projection. Especially, it is shown that the coupled states of identical bosons can always be expressed in terms of zeros of Jacobi polynomials. However, unlike non-identical particle systems, the coupled states of identical particles are non-orthogonal with respect to the multiplicity label after the projection. In order to establish orthonormalized coupled states for identical particles, the GramSchimidt process may be adopted. It will be advantageous in the application, for example, to the shell model calculations if matrix elements of one- and two-body operators under the angular momentum projected basis can be calculated easily, which seems possible as shown in [26] where explicit expressions for the expectation values of one- and two-body operators in the mean-field plus pairing model were obtained, of which the relevant research is in progress.

One of the authors (PF) is grateful to Faculty of Science, The University of Queensland, for support through an Ethel Raybould Visiting Fellowship. Support from U.S. National Science Foundation (OCI-0904874), Southeastern Universities Research Association, Natural Science Foundation of China (11175078), Australian Research Council (DP110103434), Doctoral Program Foundation of the State Education Ministry of China (20102136110002), and LSU-LNNU joint research program (9961) is acknowledged.

[1] P. O. Löwdin, Rev. Mod. Phys. 39, 966 (1964).

[2] P. Ring and P. Schuck, The Nuclear Many-body problem (Springer-Verlag, Berlin, 1980).

[3] L. C. Biedenharn and J. D. Louck, Angular Momentum in Quantum Physics, Theory and Application (AddisonWesley, Reading, MA, 1981)

[4] R. R. Whitehead, A. Watt, B. J. Cole, and I. Morrison,
Adv. Nucl. Phys. 9, 123 (1977), ed. M. Baranger and E. Vogt (Plenum, NY, 1977).

[5] J. B. French, E. C. Halbert, J. B. McGrory, S. S. M. Wong, Adv. in Nucl. Phys. 3, 193 (1969), ed. M. Baranger, E. Vogt (Plenum, NY, 1969).

[6] J. P. Elliott, Proc. Roy. Soc. London A 245, 562 (1958); Proc. Roy. Soc. London A 272, 557 (1963).

[7] K. Hara and Y. Int. J. Mod. Phys. E 04, 637 (1995).

[8] F. Pan, M.-L. Ge, and J. P. Draayer, J. Phys. A 33, 4917 (2000).

[9] X. Guan, K. D. Launey, M. Xie, L. Bao, F. Pan, and J. P. Draayer, Phys. Rev. C 86, 024313 (2012).

[10] F. Pan, L. Bao, L. Zhai, X. Cui, and J. P. Draayer, J. Phys. A: Math. Theor. 44, 395305 (2011).

[11] S. Lerma H. and J. Dukelsky, Nucl. Phys. B 870, 421 (2013).

[12] S. De Baerdemacker, Phys. Rev. C 86, 044332 (2012).

[13] I. Marquette and J. Links, J. Stat. Mech., P08019 (2012).

[14] Y.-Z. Zhang, J. Phys. A: Math. Theor. 45, 065206 (2012).

[15] G. Szegö, Amer. Math. Soc. Colloq. Publ. Vol. 23 (American Mathematical Society, Providence, RI, 1975).

[16] A. Faribault, O. El Araby, C. Sträter, and V. Gritsev, Phys. Rev. B 83, 235124 (2011).

[17] J. D. Louck and L. C. Biedenharn, Lecture Notes in Chemistry 12, 148 (1979), ed. J. Hinze (Springer, Berlin, 1979).

[18] F. Pan and J. P. Draayer, J. Math. Phys. 39, 5631 (1998).

[19] F. Pan, M.-X. Xie, X. Guan, L.-R. Dai, and J. P. Draayer, Phys. Rev. C 80, 044306 (2009).

[20] R. W. Richardson, Phys. Lett. 3, 277 (1963); 5, 82 (1963); R. W. Richardson and N. Sherman, Nucl. Phys. 52, 221 (1964); 52, 253 (1964); M. Gaudin, J. Phys. 37, 1087 (1976).

[21] M. Hamermesh, Group Theory and Its Application to Physical Problems (Addison-Wesley, London, 1962).

[22] F. Iachello, Lie Algebras and Applications, Lecture Notes in Physics 708 (Springer-Verlag, Berlin, 2006).

[23] M. A. Caprio, J. H. Skrabacz, and F. Iachello, J. Phys. A: Math. Theor. 44, 075303 (2011).

[24] B. H. Flowers, Proc. Roy. Soc. London A 212, 248 (1952).

[25] A. de-Shalit and I. Talmi, Nuclear Shell Theory, (Academic, New York, 1963) [Reprint Dover, New York, 2004].

[26] R. W. Richardson, J. Math. Phys. 6, 1034 (1965). 